\title{
Role of Genetic Polymorphisms in Therapeutic Response to Anti-Asthma Therapy
}

\author{
John Oppenheimer, MD
}

\begin{abstract}
Over the past several decades, significant advances have been seen in the diagnosis and treatment of asthma. Recent research has focused on potential phenotypic and genotypic predictors of response to therapy. In this review, we will examine each of the three major therapeutic classes of asthma therapy, focusing on a potential genetic clue to medication response.
\end{abstract}

\section{Key words: asthma, genetic polymorphisms}

$\mathrm{O}$ ur understanding of the pathophysiology and treatment of asthma is quickly advancing. Clinicians have long understood that asthmatic patients' response to therapy is quite variable. This means that there are "responders" and "nonresponders" to a specific class of asthma therapy. Several recent studies have highlighted phenotypic indicators of response. ${ }^{1,2}$ As an example, in a recent study by Szefler and colleagues, it was found that baseline characteristics associated with a good response (increase in forced expiratory volume in 1 second $\left[\mathrm{FEV}_{1}\right]>15 \%$ ) to an inhaled corticosteroid (ICS) were high exhaled nitric oxide (eNO) level, high $\beta 2$ reversibility, and a low FEV to forced vital capacity ratio. ${ }^{1}$ An exciting direction with regard to response to asthma therapy is the potential role of a patient's genetics in determining therapeutic response. Although, initially, the nomenclature associated with examining genetic polymorphisms (GPs) may seem like bringing meaning to alphabet soup, it is quite likely that further advancement in our understanding of genetics will provide the clinician with a useful tool in patient care. In the case of cancer, intensive research is ongoing regarding the evaluation of a patient's genetics to facilitate in making decisions regarding specific chemotherapy. ${ }^{3}$ It is hoped that similar genetic consideration will aid in the choice of class of asthma medication in the future. In this article, we review each of the three major therapeutic classes of asthma therapy and examine a specific GP and its contribution to response. It should be noted that

John Oppenheimer: Pulmonary and Allergy Associates, Summit, NJ; Department of Internal Medicine, New Jersey Medical School, Newark, NJ.

Correspondence to: Dr. John Oppenheimer, Pulmonary and Allergy Associates, 1 Springfield Avenue, Summit, NJ 07901; e-mail: nnilopp@ oprorline.net.

DOI 10.2310/7480.2007.00003 several GPs have been identified for each of these therapeutic classes; however, examination of all would be beyond the scope of this review.

The most extensively studied GP in therapeutic asthma response involves the $\beta_{2}$ agonist receptor. The $\beta_{2}$ receptor belongs to the $G$ protein-coupled receptor superfamily, which contains 413 amino acids and was cloned in $1987 .^{4}$ Since then, several single nucleotide polymorphisms (SNPs) have been identified, including Gly16, Ile164, and Glu27. These have been reviewed in detail elsewhere. ${ }^{5}$ The majority of studies indicate that the Gly16 SNP appears to carry the greatest potential clinical impact. The wild genetic makeup of the sixteenth amino acid position of the $\beta_{2}$ receptor is GlyGly; however, in approximately one-sixth of the North American population, Arg-Arg is seen. This SNP appears to carry significant potential clinical consequences. In a study by Martinez and colleagues, 269 children who were $\beta 2$ naive, participating in a longitudinal asthma study, had their bronchodilator response measured following the acute administration of albuterol. ${ }^{6}$ A positive response was considered a rise in $\mathrm{FEV}_{1}$ of greater than $15.3 \%$. When compared with homozygotes for Gly-16, homozygotes for Arg-16 were 5.3 times more likely to have a positive response. On the other hand, when examining the chronic administration of short-acting $\beta$ agonists (SABAs), a very different picture is seen. Israel and colleagues examined the same SNP in a group of 190 asthmatic patients who had participated in a trial examining the effects of regular versus as-needed albuterol for 16 weeks. ${ }^{7}$ When retrospectively analyzing the response based on genetic makeup, they found a small decline in morning peak expiratory flow in patients homozygous for Arg-16 who used albuterol regularly. This effect, however, was magnified during a 4-week run-out period, during which all subjects returned to using as-needed albuterol. By the end of the study, subjects who were 
homozygous for Arg-16 who had regularly used albuterol had a morning peak expiratory flow of $30.5 \pm 12.1 \mathrm{~L} / \mathrm{min}$ lower $(p=.012)$ than subjects with a similar SNP who had used albuterol on an as-needed basis. At the same time, there was no such decline in peak flows with regular use of albuterol in patients who were homozygous for Gly-16.

When the same group prospectively studied the response to chronic administration (16 weeks) of albuterol stratified by genotype, they demonstrated that whereas the Arg-Arg group suffered a small reduction, the Gly-Gly group demonstrated a rise in peak flows. ${ }^{8}$ The resultant difference was $24 \mathrm{~L} / \mathrm{min}(p=.0003)$. Similar genotypespecific attributable effects were seen in $\mathrm{FEV}_{1}$, symptom control, and use of supplemental reliever medicine.

Taylor and colleagues performed retrospective analysis of the relationship between GP of the $\beta 2$ receptor and clinical outcomes in a placebo-controlled, crossover trial examining the use of regularly scheduled albuterol or salmeterol in a group of 115 mild to moderate asthmatics (24 weeks). ${ }^{9}$ Once again, chronic use of albuterol in the Arg-Arg group demonstrated a negative effect. Specifically, exacerbation rates were higher in the Arg-Arg group when using chronic albuterol ( $p=.005)$; however, there appeared to be no such increase with chronic administration of salmeterol.

These findings appear to indicate that the SNP effect was limited to SABAs only, until a recent publication by Weschsler and colleagues indicated that long-acting $\beta$ agonists (LABAs) may also be affected by a patient's genetic makeup. ${ }^{10}$ In their study, retrospective re-evaluation of the Salmeterol or Corticosteroids (SOCS) and Salmeterol \pm Inhaled Corticosteroids (SLIC) trial data were analyzed based on GP. The SOCS study examined a group of asthmatic patients with $\mathrm{FEV}_{1}>80 \%$ who were randomized to receive salmeterol or an ICS (triamcinolone) for 16 weeks. ${ }^{11}$ In this study, they demonstrated higher exacerbation rates in the LABA versus ICS group. The SLIC study examined a group of steroid-naive asthmatics with baseline $\mathrm{FEV}_{1} \leq 80 \%$ of predicted, demonstrating the ability of LABA to reduce the dose of ICS without an increase in the exacerbation rate. ${ }^{12}$ When these studies were reanalyzed stratified to SNP, both the SOCS and SLIC trials demonstrated a significant fall in Peak Expiratory Flow Rate (PEFR) in the Arg-Arg group, whereas a rise was seen in the Gly-Gly group ( $p=.005$ and .048 , respectively).

These very interesting data should be viewed as preliminary evidence in that the study does have limitations. Beyond the fact that it is retrospective evaluation, it also involves a very small sample size. It has, however, catalyzed an ongoing prospective study employing larger numbers of subjects stratified by SNP, entitled the LARGE study (Long
Acting Beta Response by Genotype), in an attempt to answer the question of the role of SNPs and response to LABAs.

Several studies have demonstrated a distribution of therapeutic response to both ICS and leukotriene modifier (LTM). ${ }^{2,13}$ This phenomenon is exemplified in a study by Baumgartner and colleagues, who performed a 6-week Double-blind placebo control (DBPC) trial in 730 adult asthmatics comparing the effectiveness of montelukast with inhaled beclomethasone by assessing improvement in asthma control days as a surrogate to response. ${ }^{2}$ Although the authors demonstrated significant concordance in response $(89 \%)$, they also demonstrated variability in response, with a group of subjects who had no improvement with therapy.

Zeiger and colleagues showed similar variability in response to either an ICS or LTM and further assessed potential phenotypic indicators of response using asthma control days as a surrogate of response in a group of children. ${ }^{13}$ In this crossover study, they found that a greater response to ICS versus LTM was associated with elevated baseline eNO, greater use of $\beta_{2}$ agonists, and more positive skin test responses.

When one examines the literature regarding potential SNP and response to LTM, two loci are of interest. The first is 5-lipoxygenase (5-LO) promoter and the second is leukotriene $\mathrm{C}_{4}$ synthase $\left(\mathrm{LTC}_{4} \mathrm{~S}\right)$. Although there has been some interesting work demonstrating the potential import of $5 \mathrm{LO}$ SNPs, ${ }^{14}$ we will focus our discussion on $\mathrm{LTC}_{4} \mathrm{~S}$. This enzyme controls cysteinyl leukotriene biosynthesis. It exists in two common alleles distinguished by an $\mathrm{A}$ or $\mathrm{C}$ transversion at a site 444 nucleotides upstream from the translational start. In a study by Sanek and colleagues, subjects with aspirin-intolerant asthma (an entity associated with increase in leukotriene production) were more commonly of the $444 \mathrm{C}$ allele. ${ }^{15}$ This finding has not been universal, however, as a study by Kedda and colleagues was unable to demonstrate a similar association. ${ }^{16}$

To attempt to understand this SNP's association with medicine response, Sampson and colleagues examined $\mathrm{LTC}_{4} \mathrm{~S}$ SNP and clinical response to the LTM zafirlukast by examining 23 adult asthmatics who were uncontrolled despite aggressive anti-asthma therapeutic intervention. ${ }^{17}$ At baseline, all subjects were receiving ICS; 10 were receiving oral corticosteroids, 14 LABA, 4 theophylline, and 1 cyclosporine. During the study period, all subjects abstained from all medications except ICS and rescue SABA. Despite the fact that this study was of an open design as all subjects received $20 \mathrm{mg}$ of zafirlukast twice daily for 2 weeks, investigators were blinded to patient genotype. Although the results did not reach significance $(p=.1)$, they were quite provoking. The group with a wild phenotype (A/A) demonstrated an $18 \%$ reduction in $\mathrm{FEV}_{1}$, whereas the 
variant $(\mathrm{C} / \mathrm{C}$ and $\mathrm{C} / \mathrm{A})$ demonstrated a $12 \%$ rise in $\mathrm{FEV}_{1}$. Overall, this study intimates a potential role in response and reinforces the need for further large-scale prospective studies.

Corticosteroid SNPs have been the least intensively studied thus far; however, some recent data speak to potential clues in ICS response. Tantisira and colleagues investigated the genetic contribution to the variation in response to ICS therapy in asthma by assessing the association of change in lung function from candidate genes crucial to the biologic actions of corticosteroids. ${ }^{18}$ This was accomplished by retrospectively examining three independent clinical trials using ICS as the primary therapeutic intervention.

Variation in one gene, corticotrophin-releasing hormone receptor 1 (CRHR1), was consistently associated with enhanced response. In each of the studies, subjects with the GAT/GAT homozygous haplotype demonstrated greater improvement in mean $\mathrm{FEV}_{1}$ than subjects with other haplotypes. CRHR1 is the predominant corticotrophin-releasing hormone (CRH) receptor in the pituitary gland, mediating release of adrenocorticotropic hormone and the catecholaminergic response to $\mathrm{CRH}$. As decreased expression or function of CRHR1 would be expected to diminish cortisol secretion in response to inflammation, one can imagine the impact on asthma associated with polymorphisms in this gene and the subsequent greater response to exogenous corticosteroids.

Although the data presented in this review are encouraging, it is quite obvious that the consistency of the SNP effect is far from universal. These inconsistencies may be a result of the fact that not one SNP but multiple genetic loci may play a simultaneous role in response to pharmacotherapy. Future research regarding the mysteries of asthma will focus on not only phenotypic indicators but also better understanding of potential contribution of a patient's genetic secrets. Although our research in these genetic clues is primordial and unraveling this genetic mystery appears to be a daunting task, it is a worthwhile one, as once understood, we may be able to rely on a patient's genetics to aid in choosing the most appropriate therapy. As clinicians attempting to stay current in the evolving science of asthma, we must continue to follow the very exciting literature regarding potential phenotypic and genotypic indicators of response to therapy because one or the combination of both of these tools will likely be a clue to a patient's response to therapy.

\section{References}

1. Szefler S, Martin RJ, King TS, et al. Significant variability in response to inhaled corticosteroids for persistent asthma. J Allergy Clin Immunol 2002;109:410-8.
2. Baumgartner RA, Martinez G, Edelman JM, et al. Distribution of therapeutic response in asthma control between oral montelukast and inhaled beclomethasone. Eur Respir J 2003;21:123-8.

3. Fourie J, Diasio RB. Pharmacogenetics. In: Chabner BA, Longo DL, editors. Cancer chemotherapy and biotherapy: principles and practice. 4th ed. Philadelphia: Lippincott Williams and Wilkins; 2006. p. 529-48.

4. Kobilka BK, Dixon RA, Fielle HG, et al. cDNA for the human $\beta 2$ adrenergic receptor: a protein with multiple membrane-spanning domains and encoding by a gene whose chromosomal location is shared with that of the receptor for platelet-derived growth factor. Proc Natl Acad Sci U S A 1987;84:46-50.

5. Green SA, Turki J, Hall IP, Ligget SB. Implications of genetic variability of human B2-adrenergic receptor structure. Pulm Pharmacol 1995;8:1-10.

6. Martinez FD, Graves PE, Baldini M, et al. Association between genetic polymorphisms of the beta 2-adrenoreceptor and response to albuterol in children with and without a history of wheezing. J Clin Invest 1997;100:3184-8.

7. Israel E, Drazen JM, Liggett SB, et al. The effect of polymorphisms of the beta2-adrenergic receptor on the response to regular use of albuterol in asthma. Am J Respir Crit Care Med 2000;162:75-80.

8. Israel E, Chinchilli VM, Ford JG, et al. Use of regularly scheduled albuterol treatment in asthma: genotype-stratified, randomised, placebo-controlled cross-over trial. Lancet 2004;364:1505-12.

9. Taylor DR, Drazen JM, Herbison GP, et al. Asthma exacerbations during long term $\beta$-agonist use: influence of $\beta 2$ adrenoceptor polymorphism. Thorax 2000;55:762-7.

10. Weschsler ME, Lehman E, Lazarus SC, et al. $\beta$-adrenergic receptor polymorphisms and response to salmeterol. Am J Respir Crit Care Med 2006;173:519-26.

11. Lazarus S, Boushey HA, Fahy JV, et al. Long-acting $\beta_{2}$-agonist monotherapy vs continued therapy with inhaled corticosteroids in patients with persistent asthma: a randomized controlled trial. JAMA 2001;285:2583-93.

12. Lemanske RF Jr, Sorkness CA, Mauger EA, et al. Inhaled corticosteroid reduction and elimination in patients with persistent asthma receiving salmeterol: a randomized controlled trial. JAMA 2001;285:2594-603.

13. Zeiger RS, Szefler SJ, Phillips BR, et al. Repsonse profiles to fluticasone and montelukast in mild-to-moderate persistent childhood asthma. J Allergy Clin Immunol 2006;117:45-52.

14. Drazen J, Yandava C, Dube L, et al. Pharmacogenetic association between ALOX5 promoter genotype and the response to antiasthma treatment. Nat Genet 1999;22:168-70.

15. Sanak M, Pierzhalska M, Bazan-Socha S, Szczeklik A. Enhanced expression of leukotriene C4 synthase due to overactive transcription of an allelic variant associated with aspirin-intolerant asthma Am J Respir Cell Mol Bio 2000;23:290-6.

16. Kedda M, Shi J, Duffy D, et al. Characterization of two polymorphisms in the leukotriene $\mathrm{C} 4$ synthase gene in an Australian population of subjects with mild, moderate and severe asthma. J Allergy Clin Immunol 2004;1134:889-95.

17. Sampson AP, Siddiqui S, Buchanan D, et al. Variant LTC4 synthase allele modifies cysteinyl leukotriene synthesis in eosinophils and predicts clinical response to zafirlukast. Thorax 2000;55:S28-31.

18. Tantisira KG, Lake S, Silverman ES, et al. Corticosteroid pharmacogenetics: association of sequence variants in CRHR1 with improved lung function in asthmatics treated with inhaled corticosteroids. Hum Mol Genet 2004;13:1353-9. 